\title{
Pilot Testing of Assessment of Capacity for Myoelectric Control (ACMC) in Evaluating Myoelectric Hand Function in Chinese Population
}

\section{Lai $\mathrm{FH}^{1}{ }^{*}$, Yan EW${ }^{2}$, Ma BT ${ }^{3}$, Zhao $\mathrm{ZG}^{4}$ and Liu $\mathrm{T}^{4}$}

${ }^{1}$ Department of Rehabilitation Sciences, Hong Kong Polytechnic University, Hong Kong

${ }^{2}$ Department of Occupational Therapy, Kowloon Hospital, Hong Kong ${ }^{3}$ Community Psychiatric Service, Castle Peak Hospital, Hong Kong

${ }^{4}$ Department of Orthopedic and Traumatology, Sichuan Academy of Medical Science \& Sichuan Provincial People's Hospital, Hong Kong

*Corresponding author: Lai FH, Department of Rehabilitation Sciences, Hong Kong Polytechnic University, Hong Kong, Tel: 852-2766-6749; Email: frank.hy.lai@polyu.edu.hk

\section{Abstract}

Upper limb amputations cause marked functional disability and lower the individual's self-body image, with severe psychological implications. Many rehabilitation parameters are involved in the successful rehabilitation of upper limb amputations. The aim of this study was to examine the validity and reliability of the Chinese version of Assessment of Capacity for Myoelectric Control (Chinese-ACMC) in upper limb amputated subjects and with a myo-electric-powered prosthetic hand.

To validate the Chinese version of Assessment of Capacity for Myoelectric Control (Chinese-ACMC) in upper limb amputee subjects (children and adults) with a myo-electric-powered prosthetic hand. A sample of convenience sampling of 22 subjects (11 males, 11 females) with upper limb amputation and myoelectric prosthetic hands were recorded during a regular clinical visit for ACMC. Each subject was evaluated according to four criteria: (a) Upper Limb Amputation including all levels of amputation; (b) No specific pain type - no matter phantom or pain in the stump; (c) With intact cognitive function; (d) Age ranged from 12 to 40 years. With instruction, occupational therapists and prosthetic-orthotics with at least twenty years' clinical experience of myoelectric prosthesis training would conduct the 30 -items Chinese ACMC for each subject. A serial of errand tasks of activities of daily living were designed for evaluation. Individuals' ratings were repeated after 4 weeks. Through test-retest reliability, internal consistency testing, factor analysis, intra and inter factor correlation analysis. A four-factor structure, namely, "Gripping”, "Holding”, "Releasing” and "Coordinating” are identified. 
Keywords: Functional use; Measurement; Arm Prosthesis; Occupational Therapy

Abbreviations: ACMC: Assessment of Capacity for Myoelectric Control; ICC: Intra-Class Correlation Coefficient

\section{Introduction}

Upper-limb amputations are most often the result of sudden trauma to the body, although they also can be caused by malignancy, congenital deficiencies, and vascular disease [1,2]. About 16,000 new upper-limb amputations occur annually, of which only 2,000 are at or above the wrist [3]. A person faced with such a lifealtering change will have to make many physical and psychological adjustments to be able to fully participate in everyday life [4-6]. Rehabilitation intervention will vary according to individual needs [3], and phases of treatment may overlap depending on individual's progress [6]. With the professional advices from prosthetic and orthosis, prosthetic options will be discussed with the client when he or she is medically stable. They include prescriptions of a passive prosthesis, a body-powered prosthesis, a myo-electric prosthesis, a hybrid prosthesis, a taskspecific prosthesis, or consideration of not prescribing any prosthesis [7]. Many studies conducted over recent decades have looked at the influence of certain factors in the successful use of the upper limb prosthesis. These factors include level of amputation, age, level of education, and training in prosthetic use have all been shown to be factors that influence prosthetic success [4, 6-9].

Myoelectric prostheses had become more universally applicable since early 90s [10]. Many technological advances in prosthetic devices have been made in the recent years $[11,12]$. Prostheses are available with specialized attachments that allow the person with an amputation to resume or begin participation in activities of daily living, work, athletic activities, and other leisure pursuits $[11,13,14]$. A proper assessment on the use of myoelectric hand is therefore highly necessary in documenting the rehabilitation progress.

\section{Assessment of Capacity for Myoelectric Control (ACMC)}

The ACMC is scored on the basis of observations of the myoelectric prosthesis user as he or she is performing everyday tasks [15]. Any task, easy or difficult, can be used to evaluate the capacity for control, as long as the task requires active use of both hands (i.e. the unaffected hand and the prosthetic hand). During the assessment, the subjects are encouraged to accomplish the tasks spontaneously in their usual way (i.e. by using the prosthetic hand as they are used to, as an active assisting hand or as a passive support or stabilizer of objects). The occupational therapist assesses their capacity for control of their myoelectric prosthesis by rating their performances on 30 items representing different aspects of quality of myoelectric control. The 30 items in the ACMC are classified into 4 groups: (i) gripping (12 items), (ii) holding (6 items), (iii) releasing (10 items), and (iv) co-ordinating between hands (2 items). Each person's performance is rated with scores ranging from zero to 3 , where 0 means the individual is not capable, 1 means sometimes capable, capacity not established, 2 means/capable on request, and 3 means/the subject is spontaneously capable. Only those items that are observed during the test session are scored.

\section{Methods}

Development of this Chinese version of Assessment of Capacity of Myoelectric Control (Chinese-ACMC) involved several studies using the following methods: systematic literature reviews, field testing, expert panels, inter-rater reliability studies, factor analysis to determine construct validity, longitudinal studies to examine predictive validity and focus group qualitative studies to assess the clinical utility of the tool.

\section{Procedures}

Ten Chinese and Cantonese speaking people of various educational levels ranging from primary to tertiary were recruited to complete a questionnaire to assess the linguistic validity of the Chinese-ACMC. Items in the Chinese-ACMC were modified either in syntactical or structural aspects according to written comments on individual items provided by the respondents.

Five bilingual expert panel members (1 Occupational Therapist, 2 Prosthetic and Orthoist, and 2 Medical Officers) with at least 5years experience in myo-electric powered hand rehabilitation training were invited to form the panel in reviewing the Chinese-ACMC. With the use of a set of standardized questionnaire which was designed to study the adequacy, accuracy and ease of understanding of translation and face and content validity of the Chinese-ACMC. Their professional evaluation for 
each item was deconstructed to create a number of questions that could be answered as either yes or no, and to complete the questionnaire.

Each item in the Chinese-ACMC will be examined by this expert panel group. A questionnaire for evaluating the content validity of Chinese-ACMC was designed and sent together with a covering letter and both the original ACMC and the Chinese- ACMC. The professional evaluation for each item was deconstructed to create a number of questions that could be answered by subject, and can indicate their preference out from a visual analog scale.

The drafted items were pre-test with five subjects whom will be not involved in the main study. According to their suggestions the format of some questions may be changed. Convenient sampling of 22 subjects was recruited, all of them were survival from the earthquake in Mainland China. Recruited subjects should be upper limb amputee client, literate but should not have any mental function deficiency. The reviewed Chinese-ACMC were then administrated to subjects again for inter-rater reliability, each subject was verified by two examiners in assessing the same subject in the same situ. Moreover, for the test-retest reliability, each subject was asked to complete the Chinese -ACMC for two times, the interval between the two measures should be within one week. Further analysis on factor analysis, intra and inter factor correlation analysis would be conducted.

The reviewed questionnaire will be kept strictly confidential, data collected will be utilized for further testretest reliability, internal consistency, factor analysis, intra and inter factor correlation analysis.

\section{Results}

Among these five subjects in assessing the linguistic validity of Chinese - ACMC, three were female and two were male, age ranged from 10-39. The degree of clear presentation and content understandability were presented in a 0 to 10 Likert scale, and a stringent cut-off mean score $(\sim 80 \%$ agreement $)$ of clear presentation or understandability was set. The mean scores were calculated and score of eight was selected as the cut-off score of a clear presentation and good content understandability. The results were summarized in Table 1. Items with either mean score of clear presentation or understandability below the cut-off score were needed to reviewed again. 7 items $(6,10,12,18,19,22$ and 29) were refined to increase the degree of clarity, comprehensiveness, and comprehensibility. Items were minorly refined in semantics. Inter-rater reliability testing using kappa scores (kappa scores $=0.62$ ) indicated appropriate agreement between raters [16].

\begin{tabular}{|c|c|c|c|}
\hline $\begin{array}{c}\text { Item } \\
\text { No. }\end{array}$ & $\begin{array}{c}\text { Clear } \\
\text { Presentation }\end{array}$ & Understandability & $\begin{array}{c}\text { Relevancy } \\
\text { to } \\
\text { Concept }\end{array}$ \\
\hline 1 & 8.47 & 8.53 & 8.90 \\
\hline 2 & 8.38 & 8.43 & 8.70 \\
\hline 3 & 8.47 & 8.53 & 8.90 \\
\hline 4 & 8.47 & 8.53 & 8.67 \\
\hline 5 & 8.02 & 8.52 & 8.13 \\
\hline 6 & $* 6.90$ & $* 6.88$ & 8.23 \\
\hline 7 & 8.9 & 9.88 & 8.23 \\
\hline 8 & 9 & 8.85 & 8.92 \\
\hline 9 & 8.73 & 8.07 & 8.83 \\
\hline 10 & $* 7.42$ & $* 7.88$ & 8.70 \\
\hline 11 & 8.3 & 8.32 & 8.42 \\
\hline 12 & $* 7.68$ & $* 7.68$ & 8.17 \\
\hline 13 & 8.18 & 8.15 & 8.05 \\
\hline 14 & 9 & 8.85 & 8.92 \\
\hline 15 & 8.75 & 8.22 & 8.43 \\
\hline 16 & 8.35 & 8.65 & 8.45 \\
\hline 17 & 8.32 & 8.13 & 8.07 \\
\hline 18 & $* 7.68$ & $* 7.68$ & $* 7.10$ \\
\hline 19 & $* 7.17$ & $* 7.90$ & $* 7.72$ \\
\hline 20 & 8.3 & 8.32 & 8.42 \\
\hline 21 & 9.68 & 8.68 & 8.17 \\
\hline 22 & $* 7.68$ & $* 7.68$ & $* 7.10$ \\
\hline 23 & 9 & 8.85 & 8.92 \\
\hline 24 & 8.75 & 8.22 & 8.43 \\
\hline 25 & 8.35 & 8.65 & 8.45 \\
\hline 26 & 8.32 & 8.13 & 8.07 \\
\hline 27 & 9.68 & 8.68 & 8.10 \\
\hline 28 & 8.17 & 8.9 & 8.72 \\
\hline 29 & $* 7.32$ & $* 7.13$ & $* 7.07$ \\
\hline 30 & 8.23 & 8.34 & 8.09 \\
\hline
\end{tabular}

Table 1: Mean Scores of Linguistic Validity of the 30 Items of the Chinese version of Assessment of Capacity of Myoelectric Control (Chinese- ACMC).

*refer back to expert panel to review.

All five panel members completed a questionnaire, which surveyed the accuracy of translation and content validity of the Chinese- ACMC. The panel members were invited to judge whether they agreed that the translation of each item was accurate or not, by choosing "Agree" or "Disagree". Qualitative comments for modification of 
translation were also requested if "Disagree" was rated for that item. The level of agreement of the panel members on the translation of items of the Chinese-ACMC was summarized in Table 2 . The reasons for the changes could be broadly classified into 6 categories: 1) use of specific terms; 2) use of generic terms; 3) inaccurate translation; 4) simplification of sentence; 5) clarification of meaning; and 6) grammatical reason.

\begin{tabular}{|c|c|c|c|}
\hline Item No. & Agree & Disagree & \% of Agreement \\
\hline 1 & 4 & 1 & 80.0 \\
\hline 2 & 4 & 1 & 80.0 \\
\hline 3 & 5 & 0 & 100.0 \\
\hline 4 & 5 & 0 & 100.0 \\
\hline 5 & 4 & 1 & 80.0 \\
\hline 6 & 4 & 1 & 80.0 \\
\hline 7 & 4 & 1 & 80.0 \\
\hline 8 & 4 & 1 & 80.0 \\
\hline 9 & 4 & 1 & 80.0 \\
\hline 10 & 4 & 1 & 80.0 \\
\hline 11 & 4 & 1 & 80.0 \\
\hline 12 & 4 & 1 & 80.0 \\
\hline 13 & 4 & 1 & 80.0 \\
\hline 14 & 5 & 0 & 100.0 \\
\hline 15 & 5 & 0 & 100.0 \\
\hline 16 & 4 & 1 & 80.0 \\
\hline 17 & 4 & 1 & 80.0 \\
\hline 18 & 5 & 0 & 100.0 \\
\hline 19 & 5 & 0 & 100.0 \\
\hline 20 & 4 & 1 & 80.0 \\
\hline 21 & 5 & 0 & 100.0 \\
\hline 22 & 4 & 1 & 80.0 \\
\hline 23 & 4 & 1 & 80.0 \\
\hline 24 & 4 & 1 & 80.0 \\
\hline 25 & 4 & 1 & 80.0 \\
\hline 26 & 4 & 1 & 80.0 \\
\hline 27 & 4 & 1 & 80.0 \\
\hline 28 & 4 & 1 & 80.0 \\
\hline 29 & 4 & 1 & 80.0 \\
\hline 30 & 4 & 1 & 80.0 \\
\hline & & & \\
\hline
\end{tabular}

Table 2: Percentage of Agreement of the Translation of the 30 Items of the Chinese-ACMC.

The content relevancy of the Chinese - ACMC was evaluated in terms of whether the items were relevant to the capacity in myoelectric control Table 3 . The results showed that all experts considered all items as relevant to the content area. The percentage of agreement ranged from $75 \%$ to $100 \%$, and the median scores were ranged from 4 to 5 in the items. Therefore, the thirty items in the Chinese-ACMC were regarded as good items.

The content representativeness of the Chinese-ACMC was examined in terms of the thirty items as a whole to the measurement of capacity in myoelectric control. The result was summarized in Table 4 . The percentage of agreement was $100 \%$ and median score was 4 , which indicated all experts agreed that these thirty items cover the major constructs and could represent a person's capacity in myoelectric hand control.

\begin{tabular}{|c|c|c|c|c|}
\hline \multirow[b]{2}{*}{ Item } & \multicolumn{3}{|c|}{$\%$ of Agreement } & \multirow[b]{2}{*}{ Median } \\
\hline & Agree & Neutral & Disagree & \\
\hline 1 & 100 & 0 & 0 & 4.5 \\
\hline 2 & 100 & 0 & 0 & 4.5 \\
\hline 3 & 80 & 20 & 0 & 4 \\
\hline 4 & 100 & 0 & 0 & 4.5 \\
\hline 5 & 100 & 0 & 0 & 4.5 \\
\hline 6 & 100 & 0 & 0 & 4.5 \\
\hline 7 & 80 & 20 & 0 & 4.5 \\
\hline 8 & 80 & 20 & 0 & 4 \\
\hline 9 & 100 & 0 & 0 & 4.5 \\
\hline 10 & 100 & 0 & 0 & 4.5 \\
\hline 11 & 80 & 20 & 0 & 4 \\
\hline 12 & 80 & 20 & 0 & 4.5 \\
\hline 13 & 80 & 20 & 0 & 4 \\
\hline 14 & 100 & 0 & 0 & 5 \\
\hline 15 & 100 & 0 & 0 & 5 \\
\hline 16 & 100 & 0 & 0 & 4.5 \\
\hline 17 & 100 & 0 & 0 & 4 \\
\hline 18 & 80 & 20 & 0 & 4 \\
\hline 19 & 80 & 20 & 0 & 4.5 \\
\hline 20 & 80 & 20 & 0 & \\
\hline 21 & 80 & 0 & 20 & 4.5 \\
\hline 22 & 100 & 0 & 0 & 4.5 \\
\hline 23 & 80 & 20 & 0 & 4 \\
\hline 24 & 100 & 0 & 0 & 4.5 \\
\hline 25 & 100 & 0 & 0 & 4.5 \\
\hline 26 & 100 & 0 & 0 & 4.5 \\
\hline 27 & 80 & 20 & 0 & 4.5 \\
\hline 28 & 80 & 20 & 0 & 4 \\
\hline 29 & 100 & 0 & 0 & 4.5 \\
\hline 30 & 80 & 20 & 0 & 4.5 \\
\hline
\end{tabular}

Table 3: Results of Content Relevancy of the Chinese ACMC. 


\begin{tabular}{|c|c|c|c|c|}
\hline \multirow{2}{*}{} & \multicolumn{3}{|c|}{ \% of Agreement } & \\
\cline { 2 - 5 } & Agree & Neutral & Disagree & Median \\
\hline Items 1-30 & 100 & 0 & 0 & 4 \\
\hline
\end{tabular}

Table 4: Results of Content Representativeness of the Chinese-ACMC.

The inter-rater reliability was verified by two examiners in assessing the same subject in situ. The interrater reliability coefficient was .91 , with an intra-class correlation coefficient (ICC) of .92 (with individual item ICCs ranging from .86 to .95 (95\% CI: .88-.95)), and good to excellent internal consistency (Cronbach's alpha $=.92$ ). The Chinese ACMC showed good test-retest reliability, with an intra-class correlation coefficient (ICC) of .93 (with individual item ICCs ranging from .83 to .95 (95\% CI: .84-.95)), and good to excellent internal consistency (Cronbach's alpha $=.91$ ).

The highest potential source of error comes from the test-retest intervals. A one-week interval may be short, and carryover effect can occur. In practical, a very good reliability coefficient proves nearly no obvious effect from time interval. This good reliability coefficient implied nearly no obvious effect from time interval, as reflected by the nature of capacity of myoelectric control which will not be changed rapidly with time $[17,18]$.
The factor structure was examined by factor analysis. The result of the Bartlett's test of sphericity was 938.18 ( $p$ $=.04$ ), and the value of the Kaiser-Meyer-Olkin Measure of Sampling Adequacy was .703. These high values supported further application of factor analysis to explore the latent structure of the Chinese ACMC.

Principal component analysis method of factor extraction was used in this study. Table 5 reported the statistics of principal component analysis. In the initial analysis, a total of four factors could be extracted with the eigenvalue greater than 1.00. In terms total variance, four factors accounted for $50.89 \%$ variance, which can be considered as a marginally reasonable factor number. Furthermore, the scree plot in Figure 1 showed the curve straightens out at the four factors. Therefore, the fitness of four-factor solution could be justified. In the rotation phase of factor analysis, it was aimed at achieving simpler and theoretically more meaningful factor solutions. Oblimin rotation was employed in further investigating the factor loadings. The result was summarized in Table 6. The four factors were labeled according to the items' nature of construct. Factor 1 was "Gripping"; factor 2 was "Holding"; factor 3 was "Releasing"; and Factor 4 was "Coordinating".

\begin{tabular}{|c|c|c|c|c|c|}
\hline Items & Communality & Factor & Eigenvalue & \% of Variance & Cumulative \% \\
\hline 1 & .576 & 1 & 4.701 & 15.670 & 15.670 \\
\hline 2 & .434 & 2 & 3.841 & 12.803 & 28.473 \\
\hline 3 & .424 & 3 & 3.382 & 11.275 & 39.748 \\
\hline 4 & .533 & 4 & 3.324 & 11.082 & 50.829 \\
\hline 5 & .521 & 5 & 2.573 & 8.576 & 59.405 \\
\hline 6 & .506 & 6 & 2.319 & 7.730 & 67.135 \\
\hline 7 & .389 & 7 & 1.727 & 5.758 & 72.893 \\
\hline 8 & .373 & 8 & 1.601 & 5.337 & 78.230 \\
\hline 9 & .545 & 9 & 1.441 & 4.803 & 83.033 \\
\hline 10 & .523 & 10 & 1.271 & 4.237 & 91.270 \\
\hline 11 & .392 & 11 & 1.121 & 3.738 & 93.885 \\
\hline 12 & .623 & 12 & .863 & 2.878 & 96.209 \\
\hline 13 & .534 & 13 & .697 & 2.324 & 97.917 \\
\hline 14 & .655 & 14 & .512 & 1.708 & 98.750 \\
\hline 15 & .484 & 15 & .250 & .833 & 99.404 \\
\hline 16 & .762 & 16 & .196 & .654 & 99.766 \\
\hline 17 & .634 & 17 & .109 & .362 & 100.000 \\
\hline 18 & .359 & 18 & .070 & .234 & 100.000 \\
\hline 19 & .648 & 19 & $6.610 \mathrm{E}-16$ & $2.203 \mathrm{E}-15$ & 100.000 \\
\hline 20 & .392 & 20 & $3.752 \mathrm{E}-16$ & $1.251 \mathrm{E}-15$ & 100.000 \\
\hline 21 & .462 & 21 & $2.983 \mathrm{E}-16$ & $9.942 \mathrm{E}-16$ & 100.000 \\
\hline 22 & .466 & 22 & $1.291 \mathrm{E}-16$ & $4.304 \mathrm{E}-16$ & \\
\hline
\end{tabular}

Lai FH, et al. Pilot Testing of Assessment of Capacity for Myoelectric Control (ACMC) in Evaluating Myoelectric Hand Function in Chinese Population. Ann Physiother Occup 


\begin{tabular}{|l|l|l|l|l|l|}
\hline 23 & .432 & 23 & $4.316 \mathrm{E}-17$ & $1.439 \mathrm{E}-16$ & 100.000 \\
\hline 24 & .421 & 24 & $-7.176 \mathrm{E}-17$ & $-2.392 \mathrm{E}-16$ & 100.000 \\
\hline 25 & .562 & 25 & $-1.369 \mathrm{E}-16$ & $-4.564 \mathrm{E}-16$ & 100.000 \\
\hline 26 & .672 & 26 & $-2.164 \mathrm{E}-16$ & $-7.213 \mathrm{E}-16$ & 100.000 \\
\hline 27 & .762 & 27 & $-4.328 \mathrm{E}-16$ & $-1.443 \mathrm{E}-15$ & 100.000 \\
\hline 28 & .587 & 28 & $-5.528 \mathrm{E}-16$ & $-1.843 \mathrm{E}-15$ & 100.000 \\
\hline 29 & .671 & 29 & $-6.834 \mathrm{E}-16$ & $-2.278 \mathrm{E}-15$ & 100.000 \\
\hline 30 & .358 & 30 & $-1.003 \mathrm{E}-15$ & $-3.342 \mathrm{E}-15$ & 100.000 \\
\hline
\end{tabular}

Table 5: Initial Statistics for Each Factor of Chinese-ACMC.

Bartlett's test of sphericity: 938.18 ( $\mathrm{p}=.000)$

Kaiser-Meyer-Olkin Measure of Sampling Adequacy: .703

Extraction Method: Principal Component Analysis

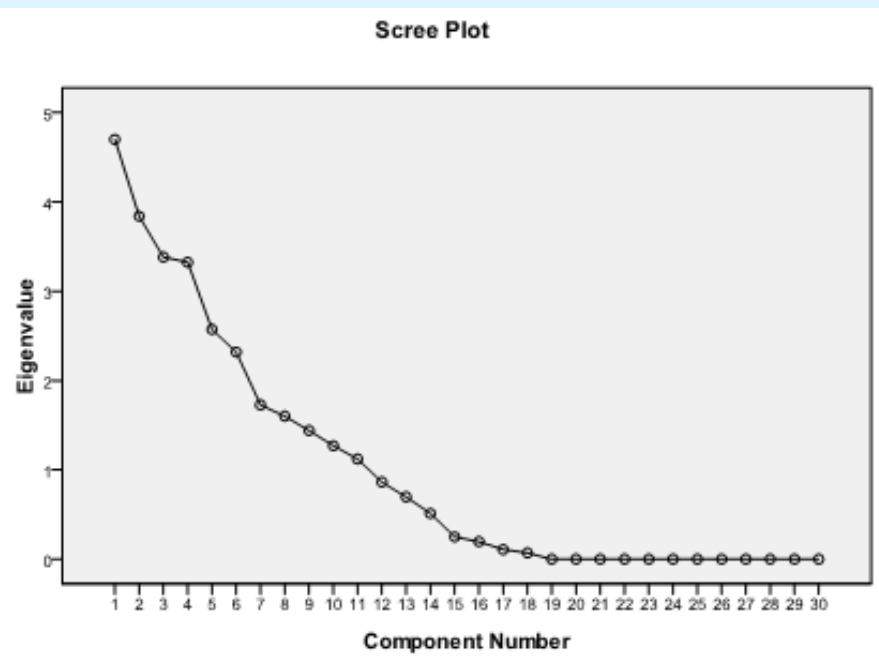

Figure 1: Scree Plot of the Factors of the Chinese-ACMC.

\begin{tabular}{|c|c|c|c|c|}
\hline Items & Factor 1 & Factor 2 & Factor 3 & Factor 4 \\
\hline 1 & .908 & & & \\
\hline 6 & .828 & & & \\
\hline 10 & .815 & & & \\
\hline 11 & .697 & & & \\
\hline 12 & .642 & & & \\
\hline 7 & .628 & & & \\
\hline 9 & .61 & & & \\
\hline 8 & .586 & & & \\
\hline 2 & .579 & & & \\
\hline 3 & .567 & & & \\
\hline 5 & .531 & & & \\
\hline 4 & .529 & & & \\
\hline 13 & & .783 & & \\
\hline 18 & & .783 & & \\
\hline
\end{tabular}




\begin{tabular}{|l|l|l|l|l|}
\hline 14 & & .709 & & \\
\hline 15 & & .655 & & \\
\hline 17 & & .634 & & \\
\hline 16 & & .515 & .789 & \\
\hline 19 & & & .695 & \\
\hline 21 & & & .595 & \\
\hline 23 & & & .535 & \\
\hline 26 & & & .525 & \\
\hline 27 & & & .515 & \\
\hline 20 & & & .513 & \\
\hline 22 & & & .495 & \\
\hline 25 & & & .465 & .565 \\
\hline 24 & & & & .595 \\
\hline 28 & & & & \\
\hline 30 & & & & \\
\hline 29 & & & & \\
\hline
\end{tabular}

Table 6: Factor Loadings of Items of the Chinese ACMC after Oblimin Rotation.

Extraction Method: Principal Component Analysis

Rotation Method: Oblimin Rotation

Remark: Only showed factor loadings above .35

The value of factor loadings was the criteria to group the items in different factors respectively. "Gripping" domain included 12 items, i.e. item 1,2,3,4,5,6,7,8,9,10,11 and 12. "Holding" domain included 6 items, i.e. item 13, $14,15,16,17$ and 18. "Releasing" domain included 10 items, i.e. item 19, 20, 21, 22, 23, 24, 25, 26, 27 and 28. "Coordinating" domain includes 2 items, i.e. item 29 and 30.

The relationships of these four factors were examined in terms of item description, internal consistency and correlation. Cronbach's alpha was used to show the internal consistency of the Chinese-ACMC Table 7. In these four factors, the ranges of item-total correlation were .58 to $.79, .46$ to $.65, .50$ to. 69 and .48 to .66 respectively as shown in Table 8 . They indicated a fair relationship of the items to their respective factors. The internal consistency of the four individual factors and the overall Chinese ACMC were found to be $.85, .83, .73, .70$ and .69 respectively. The moderate internal consistency implied that the items within each factor and the factors of the Chinese ACMC measured the same construct. Pearson correlation coefficients among the four factors were presented. All the factors were found to have good relationship with one another (ranged from $r=.56, p=.04$ to $r=.67, p=.04$ ). The factor 1 "Gripping" and factor 2 "Holding" had the highest relationship ( $r=.87, p=.04)$. In Table 9, the reliability coefficients of ICC among four factors were $.92, .90, .80, .84$ which was good and adequate evidence to substantiate the use of Chinese ACMC across different time.

\begin{tabular}{|c|c|c|}
\hline & $\begin{array}{c}\text { Cronbach's } \\
\text { Alpha }\end{array}$ & $\begin{array}{c}\text { Alpha of } \\
\text { Chinese Home } \\
\text { ACMC if } \\
\text { deleted }\end{array}$ \\
\hline Factor 1 - Gripping & .85 & .43 \\
\hline Factor 2 - Holding & .83 & .57 \\
\hline Factor 3 - Releasing & .73 & .52 \\
\hline $\begin{array}{c}\text { Factor 4 - } \\
\text { Coordinating }\end{array}$ & .70 & .52 \\
\hline Overall & .69 & \\
\hline
\end{tabular}

Table 7: Internal consistency of factors in Chinese ACMC.

\begin{tabular}{|c|c|c|c|c|}
\hline & Gripping & Holding & Releasing & Coordinating \\
\hline \multirow{2}{*}{ Gripping } & - & $r=.87$ & $r=.58$ & $r=.66$ \\
& & $p<.01$ & $p<.01$ & $p<.01$ \\
\hline \multirow{2}{*}{ Holding } & & - & $\begin{array}{c}r=.79 \\
p<.01\end{array}$ & $\begin{array}{c}r=.70 \\
p<.01\end{array}$ \\
\hline \multirow{2}{*}{ Releasing } & & & - & $\begin{array}{l}r=.66 \\
p<.01\end{array}$ \\
\hline \multirow{2}{*}{ Coordinating } & & & - & - \\
\hline
\end{tabular}

Table 8: Correlations among factors in Chinese-ACMC. 


\section{Annals of Physiotherapy \& Occupational Therapy}

\begin{tabular}{|c|c|}
\hline & Reliability Coefficient \\
\hline Factor 1 - Gripping & .92 \\
\hline Factor 2 - Holding & .90 \\
\hline Factor 3 - Releasing & .80 \\
\hline Factor 4 - Coordinating & .80 \\
\hline Overall Chinese ACMC & .84 \\
\hline
\end{tabular}

Table 9: Reliability coefficient of factors in Chinese-ACMC.

\section{Discussion}

The Chinese ACMC provided a structure framework for rehabilitation professionals to note and to follow what would be the essential elements in myoelectric hand rehabilitation. It is very important as a solid guideline [10] can help the rehabilitation professionals in Mainland China to organize and plan their training subsequently [13].

Provided a base for further development on psychometric properties. A four-factors structure consists of gripping, holding, releasing and coordinating are identified. These identified factors in usage as contextual free that Chinese-ACMC can be used to assess different functional tasks with the use of myoelectric hand [19]. Moreover, this type of standardized activities can help the development of service for rehabilitation starter as in Mainland China [13].

Given the normal changes associated with aging, acute illness, and deterioration in chronic diseases can contribute to a decline in individuals' ability to perform the tasks necessary to live independently in the community. With the help of this Chinese-ACMC assessment, healthcare professionals can design proper care plans for individuals in using myoelectric hands [6]. Specific identified deficits and potential assets can assist different healthcare disciplines in vocational rehabilitation planning for them.

The advantages of the Chinese ACMC assessment include the fact that it is based on the method of performance of functional tasks. This may lead to proper estimation of individual's ability. The one-week test-retest interval could be enlarged to two to three weeks in order to alleviate the memory and learning effect. In addition, the Chinese ACMC assessment may not be sensitive to small and subtle incremental changes in function. Further study can address to this gap in order to further enhance the quality of the Chinese ACMC assessment.

\section{Conclusion}

The Chinese ACMC was developed to measure the use of myoelectric hand of an upper limb amputee. The content relevancy and representativeness have been investigated with a high level of agreement. The testretest reliability was estimated to be good in terms of ICC. The construct validity is analyzed by factor analysis. A four-factor structure is extracted. The factor loading of items is ranged from .51 to .91. The names of these three factors are gripping, holding, releasing and coordinating. A moderate internal consistency is found between items, and the correlations between factors are considerably to be good. A confirmatory factor analysis across different subject groups is recommended in further study to increase the clinical utilities of Chinese ACMC. The evidences of some psychometric properties have been collected, but further research with large sample size on criterion-related validity and improvement of factor stability are recommended.

\section{References}

1. Desmond DM, Maclachlan M (2010) Prevalence and characteristics of phantom limb pain and residual limb pain in the long term after upper limb amputation. Int J Rehabil Res 33(3): 279-282.

2. Hermansson LM (1991) Structured training of children fitted with myoelectric prostheses. Prosthet Orthot Int 15(2): 88-92.

3. Ostlie K, Granan LP (2017) Guidelines for rehabilitation after acquired upper limb amputation. Tidsskr Nor Laegeforen 137(1): 15.

4. Pinzur MS, Angelats J, Light TR, Izuierdo R, Pluth T, et al. (1994) Functional outcome following traumatic upper limb amputation and prosthetic limb fitting. J Hand Surg Am 19(5): 836-839.

5. Saradjian A, Thompson AR, Datta D (2008) The experience of men using an upper limb prosthesis following amputation: positive coping and minimizing feeling different. Disabil Rehabil 30(11): 871-883.

6. Nimhurchadha S, Gallagher P, Maclachlan M, Wegener ST (2013) Identifying successful outcomes and important factors to consider in upper limb amputation rehabilitation: an international webbased Delphi survey. Disabil Rehabil 35(20): 17261733. 


\section{Annals of Physiotherapy \& Occupational Therapy}

7. Toledo C, Leija L, Munoz R, Vera A (2010) Tracking training evolution: patient with upper limb amputation above the elbow. Conf Proc IEEE Eng Med Biol Soc 2010: 2259-2262.

8. Lindner HY, Natterlund BS, Hermansson LM (2010) Upper limb prosthetic outcome measures: review and content comparison based on International Classification of Functioning, Disability and Health. Prosthet Orthot Int 34(2): 109-128.

9. Davidson JH, Khor KE, Jones LE (2010) A crosssectional study of post-amputation pain in upper and lower limb amputees, experience of a tertiary referral amputee clinic. Disabil Rehabil 32(22): 1855-1862.

10. Hermansson LM, Bodin L, Eliasson AC (2006) Intraand inter-rater reliability of the assessment of capacity for myoelectric control. J Rehabil Med 38(2): 118-123.

11. Heger H, Millstein S, Hunter GA (1985) Electrically powered prostheses for the adult with an upper limb amputation. J Bone Joint Surg Br 67(2): 278-281.

12. Guo X, Lin Z, Lyu Y, Bekrater-Bodmann R, Flor H, et al. (2017) The Effect of Prosthesis Use on Hand Mental Rotation After Unilateral Upper-Limb Amputation. IEEE Trans Neural Syst Rehabil Eng 25(11): 20462053.

13. Lindner HY, Eliasson AC, Hermansson LM (2013) Influence of standardized activities on validity of
Assessment of Capacity for Myoelectric Control. J Rehabil Res Dev 50(10): 1391-1400.

14. Kejlaa GH (1992) The social and economic outcome after upper limb amputation. Prosthet Orthot Int 16(1): 25-31.

15. Hermansson LM, Fisher AG, Bernspang B, Eliasson AC (2005) Assessment of capacity for myoelectric control: a new Rasch-built measure of prosthetic hand control. J Rehabil Med 37(3): 166-171

16. Fleiss JL, Chilton NW (1983) The measurement of interexaminer agreement on periodontal disease. J Periodontal Res 18(6): 601-606.

17. Ottenbacher KJ, Branch LG, Ray L, Gonzales VA, Peek MK, et al. (2002) The reliability of upper- and lowerextremity strength testing in a community survey of older adults. Arch Phys Med Rehabil 83(10): 14231427.

18. Ottenbacher ME, Kuo YF, Ostir GV (2007) Test-retest reliability of a psychological well-being scale in hospitalized older adults. Aging Clin Exp Res 19(5): 424-429.

19. Lindner HY, Langius-Eklof A, Hermansson LM (2014) Test-retest reliability and rater agreements of assessment of capacity for myoelectric control version 2.0. J Rehabil Res Dev 51(4): 635-644. 